\title{
Spectral Biclustering of Microarray Data: Coclustering Genes and Conditions
}

\author{
Yuval Kluger, ${ }^{1,2}$ Ronen Basri, ${ }^{3}$ Joseph T. Chang, ${ }^{4}$ and Mark Gerstein ${ }^{2,5,6}$ \\ ${ }^{1}$ Department of Genetics, Yale University, New Haven, Connecticut 06520, USA; ${ }^{2}$ Department of Molecular Biophysics \\ and Biochemistry, Yale University, New Haven, Connecticut 06520, USA; ${ }^{3}$ Department of Computer Science and Applied \\ Mathematics, Weizmann Institute of Science, Rehovot 76100, Israel; ${ }^{4}$ Department of Statistics, Yale University, New Haven, \\ Connecticut 06520, USA; ${ }^{5}$ Department of Computer Science, Yale University, New Haven, Connecticut 06520, USA
}

\begin{abstract}
Global analyses of RNA expression levels are useful for classifying genes and overall phenotypes. Often these classification problems are linked, and one wants to find "marker genes" that are differentially expressed in particular sets of "conditions." We have developed a method that simultaneously clusters genes and conditions, finding distinctive "checkerboard" patterns in matrices of gene expression data, if they exist. In a cancer context, these checkerboards correspond to genes that are markedly up- or downregulated in patients with particular types of tumors. Our method, spectral biclustering, is based on the observation that checkerboard structures in matrices of expression data can be found in eigenvectors corresponding to characteristic expression patterns across genes or conditions. In addition, these eigenvectors can be readily identified by commonly used linear algebra approaches, in particular the singular value decomposition (SVD), coupled with closely integrated normalization steps. We present a number of variants of the approach, depending on whether the normalization over genes and conditions is done independently or in a coupled fashion. We then apply spectral biclustering to a selection of publicly available cancer expression data sets, and examine the degree to which the approach is able to identify checkerboard structures. Furthermore, we compare the performance of our biclustering methods against a number of reasonable benchmarks (e.g., direct application of SVD or normalized cuts to raw data).
\end{abstract}

\section{Microarray Analysis to Classify Genes and Phenotypes}

Microarray experiments for simultaneously measuring RNA expression levels of thousands of genes are becoming widely used in genomic research. They have enormous promise in such areas as revealing function of genes in various cell populations, tumor classification, drug target identification, understanding cellular pathways, and prediction of outcome to therapy (Brown and Botstein 1999; Lockhart and Winzeler 2000). A major application of microarray technology is gene expression profiling to predict outcome in multiple tumor types (Golub et al. 1999). In a bioinformatics context, we can apply various data-mining methods to cancer datasets in order to identify class distinction genes and to classify tumors. A partial list of methods includes: (1) data preprocessing (background elimination, identification of differentially expressed genes, and normalization); (2) unsupervised clustering and visualization methods (hierarchical, SOM, k-means, and SVD); (3) supervised machine learning methods for classification based on prior knowledge (discriminant analysis, support-vector machines, decision trees, neural networks, and k-nearest neighbors); and (4) more ambitious genetic network models (requiring large amounts of data) that are designed to discover biological pathways using such approaches as pairwise interactions, continuous or Boolean networks (based on a system of coupled differential equations), and probabilistic

${ }^{6}$ Corresponding author.

E-MAIL genomeresearch@bioinfo.mbb.yale.edu; FAX (360) 8387861.

Article and publication are at http://www.genome.org/cgi/doi/10.1101/ gr.648603. graph modeling based on Bayesian networks (Tamayo et al. 1999; Brown et al. 2000; Friedman et al. 2000).

Our focus here is on unsupervised clustering methods. Unsupervised techniques are useful when labels are unavailable. Examples include attempts to identify (yet unknown) subclasses of tumors, or work on identifying clusters of genes that are coregulated or share the same function (Brown et al. 2000; Mateos et al. 2002). Unsupervised methods have been successful in separating certain types of tumors associated with different types of leukemia and lymphoma (Golub et al. 1999; Alizadeh et al. 2000; Klein et al. 2001). However, unsupervised (and even supervised) methods have had less success in partitioning the samples according to tumor type or outcome in diseases with multiple subclassifications (Pomeroy et al. 2002; van't Veer et al. 2002). In addition, the methods we propose here are related to a method of Dhillon (2001) for coclustering of words and documents.

\section{Checkerboard Structures of Genes and Conditions in Microarray Datasets}

As a starting point in analyzing microarray cancer datasets, it is worthwhile to appreciate the assumed structure of these data (e.g., whether they can be organized in a checkerboard pattern), and to design a clustering algorithm that is suitable for this structure. In particular, in analyzing microarray cancer data sets we may wish to identify both clusters of genes that participate in common regulatory networks and clusters of experimental conditions associated with the effects of these genes, for example, clusters of cancer subtypes. In both cases we may want to use similarities between expression level patterns to determine clusters. Clearly, advance knowledge of 
clusters of genes can help in clustering experimental conditions, and vice versa. In the absence of knowledge of gene and condition classes, it would be useful to develop partitioning algorithms that find latent classes by exploiting relations between genes and conditions. Exploiting the underlying twosided data structure could help the simultaneous clustering, leading to meaningful gene and experimental condition clusters.

The raw data in many cancer gene-expression datasets can be arranged in a matrix form as schematized in Figure 1. In this matrix, which we denote by $A$, the genes index rows $i$ and the different conditions (e.g., different patients) index the columns $j$. Depending on the type of chip technology used, a value in this matrix $A_{\mathrm{ij}}$ could either represent absolute expression levels (such as from Affymetrix GeneChips) or relative expression ratios (such as from cDNA microarrays). The methodology we will construct will apply equally well in both contexts. However, for clarity in what follows, we will assume that the values $A_{\mathrm{ij}}$ in the matrix represent absolute levels and that all entries are non-negative; in our numerical analyses we removed genes that did not satisfy this criterion.

A specific assumption in tumor classification is that samples drawn from a population containing several tumor types have similar expression profiles if they belong to the same type. Observing several experiments, each of which has multiple tumor types, suggests a somewhat stronger assumption; for tumors of the same type there exist subsets of overexpressed (or underexpressed) genes that are not similarly overexpressed (or underexpressed) in another tumor type. Under this assumption, the matrix $A$ could be organized in a checkerboard-like structure with blocks of high-expression levels and low-expression levels, as shown in Figure 1. A block of high-expression levels corresponds to a subset of genes (subset of rows) that are highly expressed in all samples of a given tumor type (subset of columns). One of the numerous examples supporting this picture is the CNS embryonal tumors dataset (Pomeroy et al. 2002). However, this simple checkerboard-like structure can be confounded by a number of effects. In particular, different overall expression levels of genes across all experimental conditions or of samples across all genes in multiple tumor datasets can obscure the block structure. Consequently, rescaling and normalizing both the gene and sample dimensions could improve the clustering and reveal existing latent variables in both the gene and tumor dimensions.

\section{Uncovering Checkerboard Structures Through Solving an Eigenproblem}

In this work, we attempt to simultaneously cluster genes and experimental conditions with similar expression profiles (i.e. to "bicluster" them), examining the extent to which we are able to automatically identify checkerboard structures in cancer datasets. Further, we integrate biclustering with careful normalization of the data matrix in a spectral framework model. This framework allows us to use standard linear algebra manipulations, and the resulting partitions are generated using the whole dataset in a global fashion. The normalization step, which eliminates effects such as differences in experimental conditions and basal expression levels of genes, is designed to accentuate biclusters if they exist.

Figure 1 illustrates the overall idea of our approach. It shows how applying a checkerboard-structured matrix A to a step-like classification vector for genes (x) results in a step-like classification vector on conditions (y). Reapplying the transpose of the matrix $A^{T}$ to this condition classification vectors results in a step-like gene classification vector with the same step pattern as input vector $\mathrm{x}$. This suggests that one might be able to ascertain the checkerboard-like structure of A through solving an eigenproblem involving $A A^{T}$. More precisely, it shows how the checkerboard pattern in a data matrix $A$ is reflected in the piecewise constant structures of some pair of eigenvectors $x$ and $y$ that solve the coupled eigenvalue problems $A^{T} A x=\lambda^{2} x$ and $A A^{T} y=\lambda^{2} y$ (where $x$ and $y$ have a common eigenvalue). This, in turn, is equivalent to finding the singular value decomposition of $A$. Thus, the simple operation of identifying whether there exists a pair of piecewise constant eigenvectors allows us to determine whether the data have a checkerboard pattern. Simple reshuffling of rows and columns (according to the sorted order of these eigenvectors) then can make the pattern evident. However, different average amounts of expression associated with particular genes or conditions can obscure the checkerboard pattern. This can be corrected by initially normalizing the data matrix $A$. We propose a number of different schemes, all built around the idea of putting the genes on the same scale so that they have the same average level of expression across conditions, and likewise for the conditions. A graphic overview of our method (in application to real data) is shown in Figure 8, where one can see how the data in matrix A are progressively transformed by normalization and shuffling to bring out a checkerboard-like signal.

We note that our method implicitly exploits the effect of clustering of experimental conditions on clustering of the genes and vice versa, and it allows us to simultaneously identify and organize subsets of genes whose expression levels are correlated and subsets of conditions whose expression level profiles are correlated.

\section{METHODS}

\section{Technical Background}

\section{Data normalization}

Preprocessing of microarray data often has a critical impact on the analysis. Several preprocessing schemes have been proposed. For instance, Eisen et al. (1998) prescribes the following series of operations: Take the log of the expression data, perform 5-10 cycles of subtracting either the mean or the median of the rows (genes) and columns (conditions), and then do 5-10 cycles of row-column normalization. In a similar fashion, Getz et al. (2000) first rescale the columns by their means and then standardize the rows of the rescaled matrix. The motivation is to remove systematic biases in expression ratios or absolute values that are the result of differences in RNA quantities, labeling efficiency and image acquisition parameters, as well as adjusting gene levels relative to their average behavior. Different normalization prescriptions could lead to different partitions of the data. Choice of a normalization scheme that is designed to emphasize underlying data structures or is rigorously guided by statistical principles is desirable for establishing standards and for improving reproducibility of results from microarray experiments.

\section{Singular Value Decomposition (SVD)}

Principal component analysis (PCA; Pearson 1901) is widely used to project multidimensional data to a lower dimension. PCA determines whether we can comprehensively present 


\section{(A) The Problem: Identifying Marker Genes Associated with Certain Conditions}

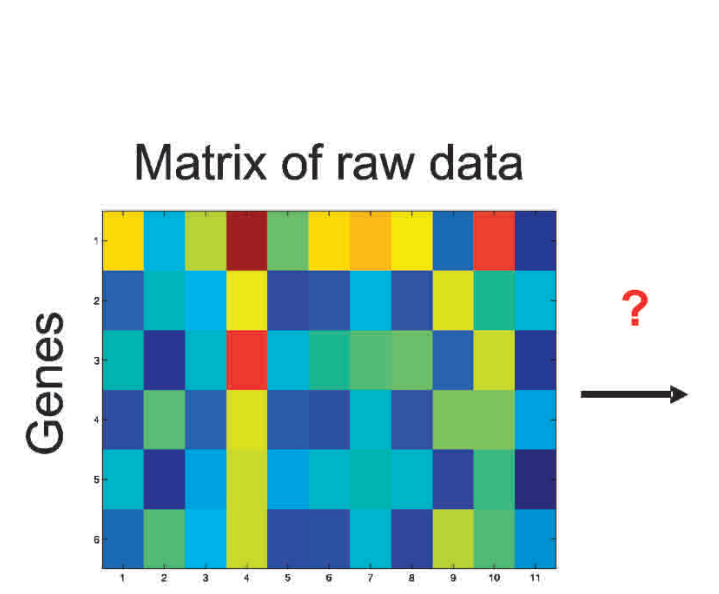

Conditions
Shuffled Matrix

(containing checkerboard "biclusters" of conditions with marker genes)

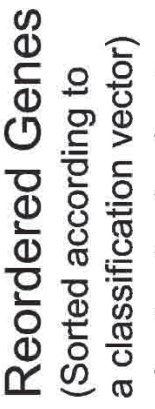

Reordered Conditions

(Sorted according to a classification vector)

\section{(B) Identifying checkerboard matrices by their action on classification vectors: Formulation as "eigenproblem"}

Gene Classification Vector $\boldsymbol{y}$

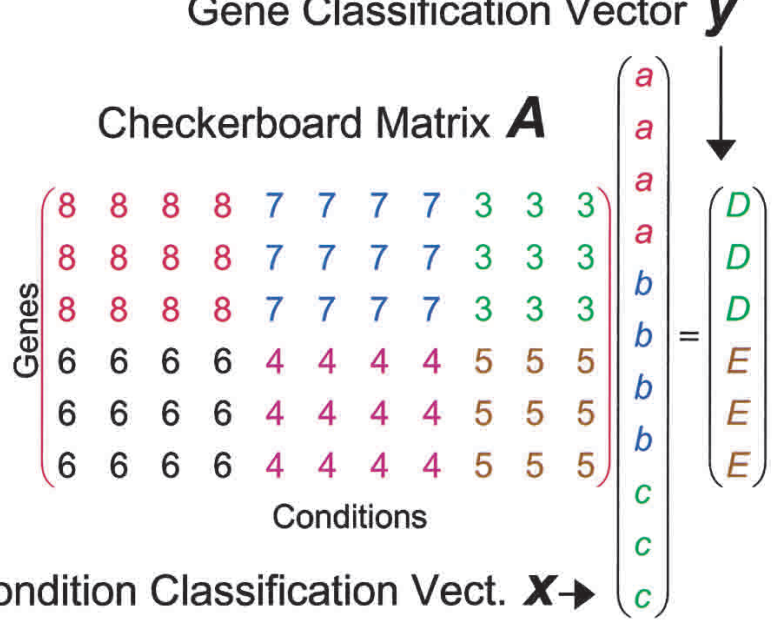

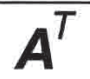

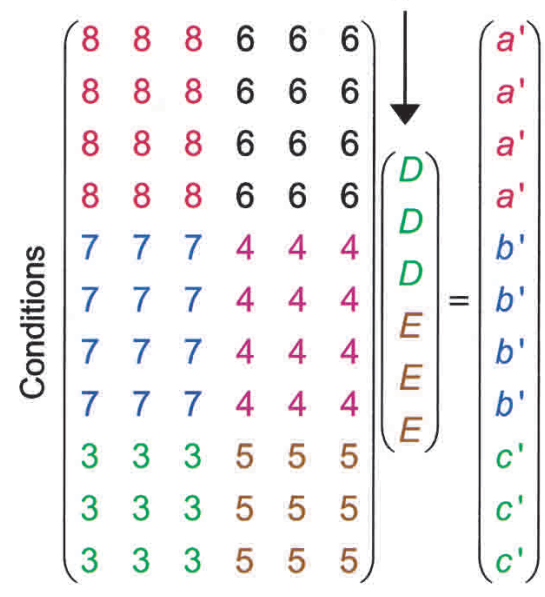

Genes
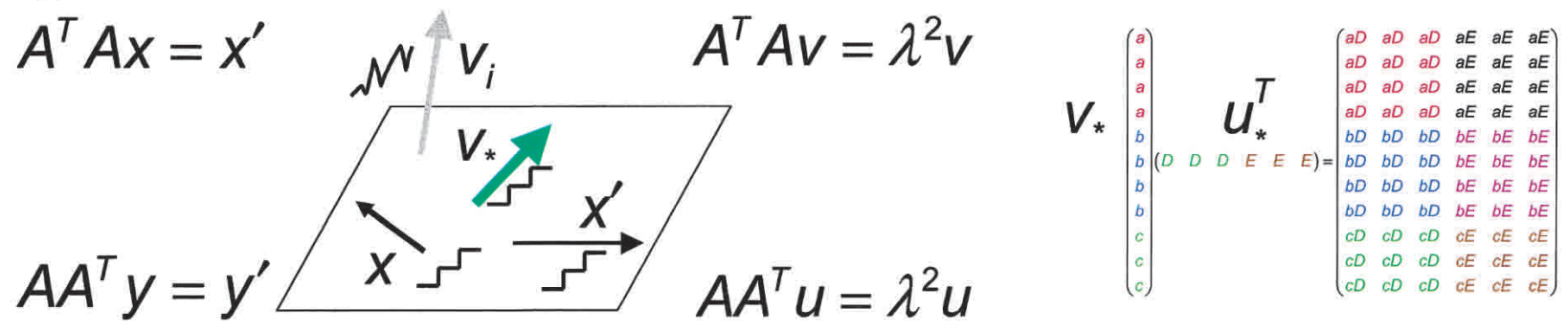

\footnotetext{
Figure 1 (Continued on next page)
} 
Kluger et al.

\section{(C) A First Step of Matrix Normalization: Rescaling Rows to Same Mean}

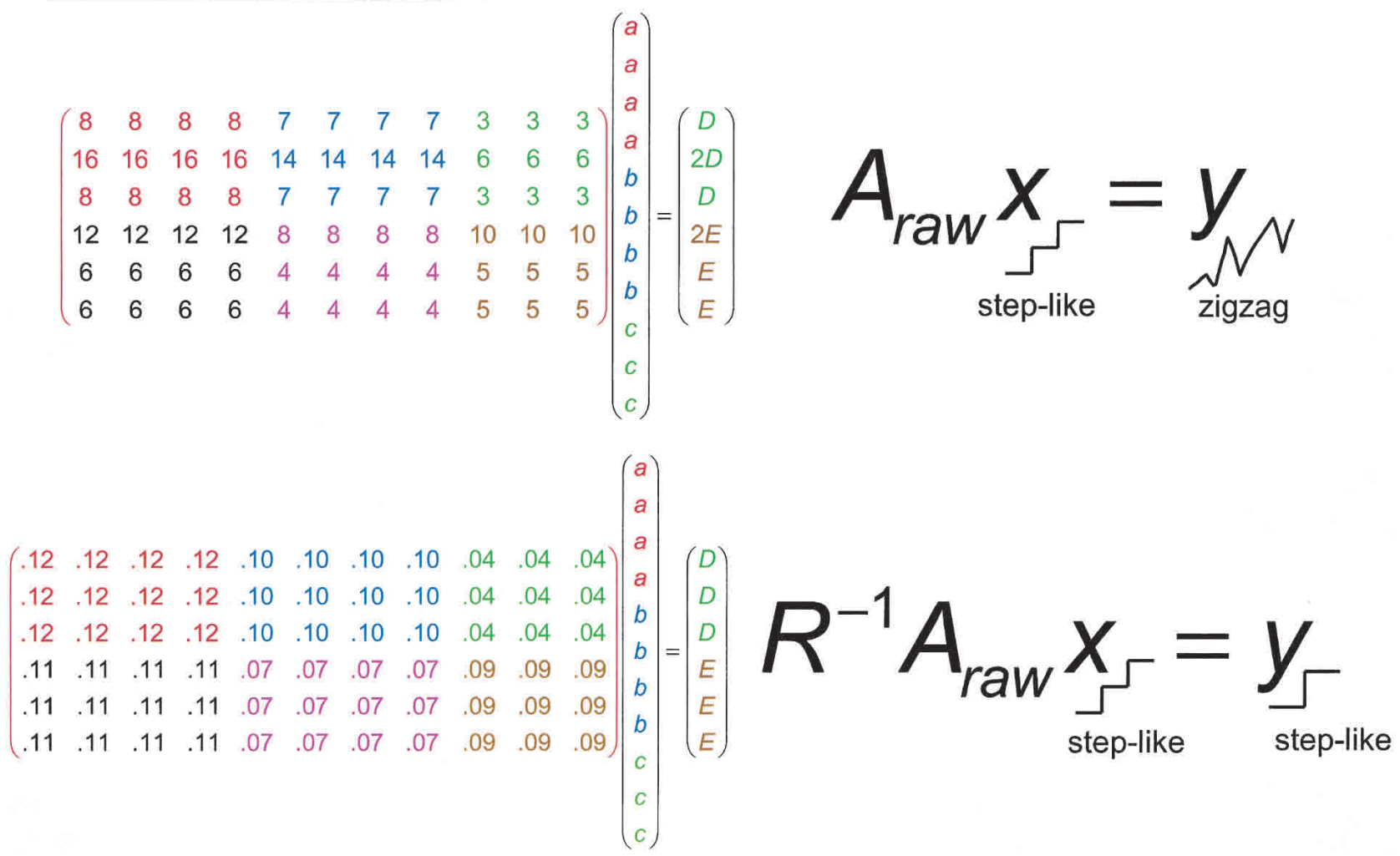

Figure 1 Overview of important parts of the biclustering process. (A) shows the problem: shuffling a gene expression matrix to reveal a checkerboard pattern associating genes with conditions. (B) shows how this problem can be approached through solving an "eigenproblem." If a gene expression matrix $A$ has a checkerboard structure, applying it to a step-like condition classification vector $x$ will result in a step-like gene classification vector $y$. Moreover, if one then applies $A^{T}$ to $y$, one will regenerate a step-like condition classification vector with the same partitioning structure as $x$. This suggests one can determine whether $A$ has a checkerboard structure through solving an eigenvalue problem. In other words, if $A$ has a (hidden) checkerboard structure, there exist some piecewise constant partition vectors $x=V_{*}$ and $y=u_{*}$ such that $A^{T} A v_{*}=\lambda^{2} v_{*}$ and $A A T u_{*}=\lambda^{2} u_{*}$ (bottom quadrant of part $B$ ). Note that most eigenvectors $v$ of the eigenvalue problem $A^{T} A v=\lambda^{2} v$ (symbolized by a zigzag structure) are not embedded in the subspace of classification (step-like) vectors $x$ possessing the same partitioning structure, as indicated by a gray arrow protruding from this subspace (parallelogram). On the other hand, piecewise constant (step-like) partition eigenvectors $v_{*}$ are embedded in this subspace and are indicated by a green arrow. To reveal whether the data have a checkerboard structure, one can inspect whether some of the pairs of monotonically sorted gene and tumor eigenvectors $v_{i}$ and $u_{i}$ have an approximate stepwise (piecewise) constant structure. The outer product $u_{*} v_{*}{ }^{T}$ of the sorted partitioning eigenvectors gives a checkerboard structure. (C) shows how rescaling of matrix $A$ can lead to improved copartitioning of genes and conditions.

multidimensional data in $d$ dimensions by inspecting whether $d$ linear combinations of the variables capture most of the data variability. The principal components can be derived by using singular value decomposition, or "SVD" (Golub and Van Loan 1983), a standard linear algebra technique that expresses a real $n \times m$ matrix $A$ as a product $A=U \Lambda V^{T}$, where $\Lambda$ is a diagonal matrix with decreasing non-negative entries, and $U$ and $V$ are $n \times \min (n, m)$ and $m \times \min (n, m)$ orthonormal column matrices. The columns of the matrices $U$ and $V$ are eigenvectors of the matrices $A A^{T}$ and $A^{T} A$, respectively, and the nonvanishing entries $\lambda_{1} \geq \lambda_{2} \geq \ldots>0$ in the matrix $\Lambda$ are square roots of the non-zero eigenvalues of $A A^{T}$ (and also of $A^{T} A$ ). Below we will denote the $i$ th columns of the matrices $U$ and $V$ by $u_{i}$ and $v_{i}$, respectively. The vectors $u_{i}$ and $v_{i}$ are called the singular vectors of $A$, and the values $\lambda_{i}$ are called the singular values. The SVD has been applied to microarray experiment analysis in order to find underlying temporal and tumor patterns (Alter et al. 2000; Holter et al. 2000; Raychaudhuri et al. 2000; Lian et al. 2001).

\section{Normalized Cuts Method}

Spectral methods have been used in graph theory to design clustering algorithms. These algorithms were used in various fields (Shi and Malik 1997), including for microarray data partitioning (Xing and Karp 2001). A commonly used variant is called the normalized cuts algorithm. In this approach the items (nodes) to be clustered are represented as the vertex set $V$. The degree of similarity (affinity) between each two nodes is represented by a weight matrix $w_{i j}$. For example, the affinity between two genes may be defined based on the correlation between their expression profiles over all experiments. The vertex set $V$ together with the edges $e_{i j} \in E$ and their corresponding weights $w_{i j}$ define a complete graph $G(V, E)$ that we want to segment. Clustering is achieved by solving an eigen- 
a

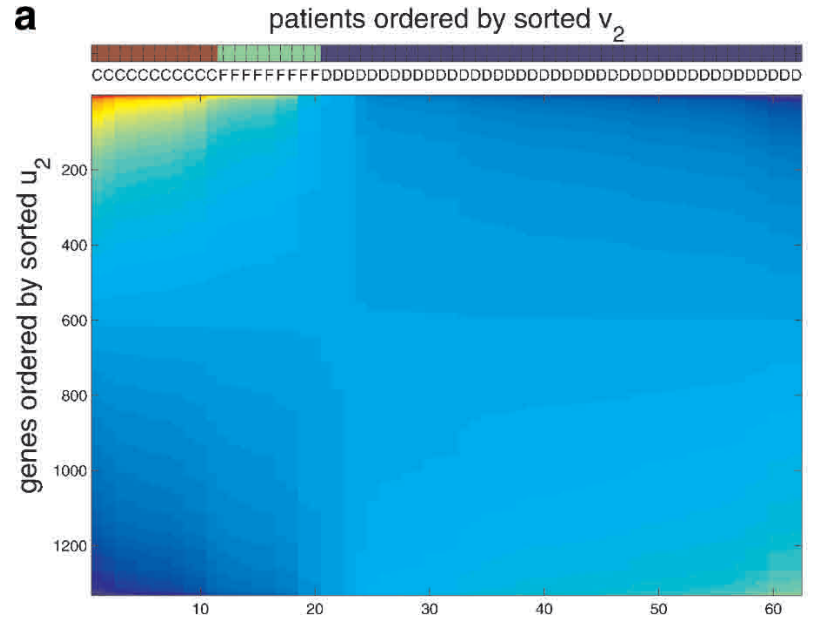

b

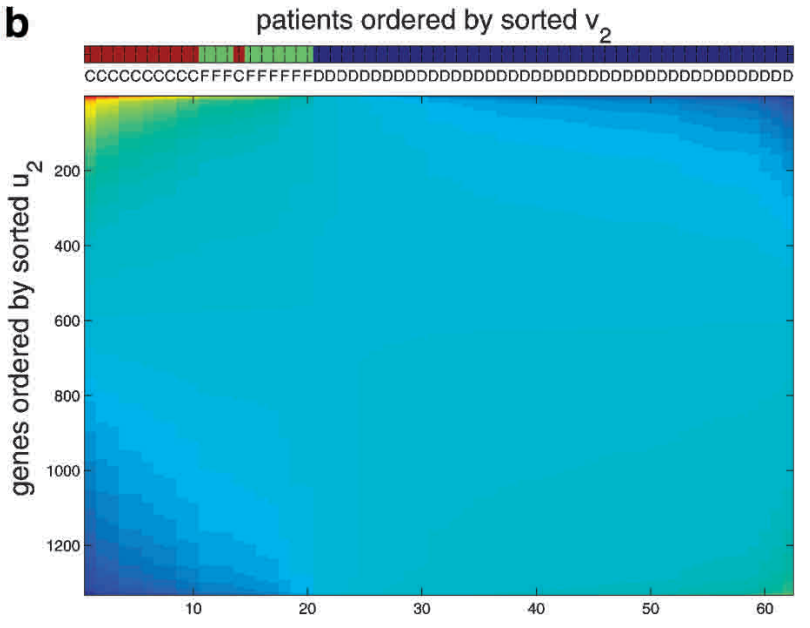

Figure 2 (a) The outer product of the sorted eigenvectors $u$ and $v$ of the 2 nd eigenvalue of the equal row- and column-sum bistochastic-like matrix $B$ applied to a dataset with three types of lymphoma: CLL (C), FL (F), and DLCL (D). Sorting of $v$ orders the patients according to the different diseases. $(b)$ As in $(a)$, the 2 nd singular value contribution to the biclustering method ( $\left.C^{-1} A^{\top} R^{-1} A\right)$ of lymphoma $C L L(C)$, $\mathrm{FL}(\mathrm{F})$, DLCL (D) partitioned the patients according to their disease, with one exception. We preselected all genes that had complete data along all experimental conditions (samples).

system that involves the affinity matrix. These methods were applied in the field of image processing, and have demonstrated good performance in problems such as image segmentation. Nevertheless, spectral methods in the context of clustering are not well understood (Weiss 1999). We note that the singular values of the original dataset represented in the matrix $A$ are related to the eigenvalues or generalized eigenvalues of the affinity matrices $A^{T} A$ and $A A^{T}$. These matrices represent similarities between genes and similarities between conditions, respectively.

\section{Previous Work on Biclustering}

The idea of simultaneous clustering of rows and columns of a matrix goes back to (Hartigan 1972). Methods for simultaneous clustering of genes and conditions were more recently proposed (Cheng and Church 2000; Getz et al. 2000; Lazzeroni and Owen 2002). The goal was to find homogeneous submatrices or stable clusters that are relevant for biological processes. These methods apply greedy iterative search to find interesting patterns in the matrices, an approach that is also common in one-sided clustering (Hastie et al. 2000; Stolovitzky et al. 2000). In contrast, our approach is more "global," finding biclusters using all columns and rows.

Another statistically motivated biclustering approach has been tested for collaborative filtering of nonbiological data (Ungar and Foster 1998; Hofmann and Puzicha 1999). In this approach, probabilistic models were proposed in which matrix rows (genes in our case) and columns (experimental conditions) are each divided into clusters, and there are link probabilities between these clusters. These link probabilities can describe the association between a gene cluster and an experimental condition cluster, and can be found by using iterative Gibbs sampling and approximated Expectation Maximization algorithms (Ungar and Foster 1998; Hofmann and Puzicha 1999).

\section{A Spectral Approach to Biclustering}

Our aim is to have coclustering of genes and experimental conditions in which genes are clustered together if they exhibit similar expression patterns across conditions and, likewise, experimental conditions are clustered together if they include genes that are expressed similarly. Interestingly, our model can be reduced to the analysis of the same eigensystem derived in Dhillon's formulation for the problem of coclustering of words and documents (Dhillon 2001). To apply Dhillon's method to microarray data, one can construct a bipartite graph, where one set of nodes in this graph represents the genes, and the other represents experimental conditions. An arc between a gene and condition represents the level of overexpression (or underexpression) of this gene under this condition. The bipartite approach is limited in that it can only divide the genes and conditions into the same number of clusters. This is often impractical. As described below, our formulation allows the number of gene clusters to be different from the number of condition clusters.

In addition, Dhillon's optimal partitioning eigenvector has a hybrid structure containing both gene and condition entries, whereas in our approach we search for separate piecewise constant structure of the gene and corresponding sample eigenvectors. Examining Dhillon's and our partitioning approaches using data generated by the generating model discussed below shows the advantage of the latter.

\section{Spectral Biclustering}

We developed a method that simultaneously clusters genes and conditions. The method is based on the following two assumptions:

1. Two genes that are coregulated are expected to have correlated expression levels, which might be difficult to observe due to noise. We can obtain better estimates of the correlations between gene expression profiles by averaging over different conditions of the same type.

2. Likewise, the expression profiles for every two conditions of the same type are expected to be correlated, and this correlation can be better observed when averaged over sets of genes of similar expression profiles.

These assumptions are supported by simple analyses of a variety of typical microarray sets. For example, Pomeroy et al. (2002) presented a dataset on five types of brain tumors, and then used a supervised learning procedure to select genes that were highly correlated with class distinction. They based this work on the absolute expression levels of genes in 42 samples taken from these five types of tumors. Using these data, we measured the correlation between the expression levels of genes that are highly expressed in only one type of tumor, 

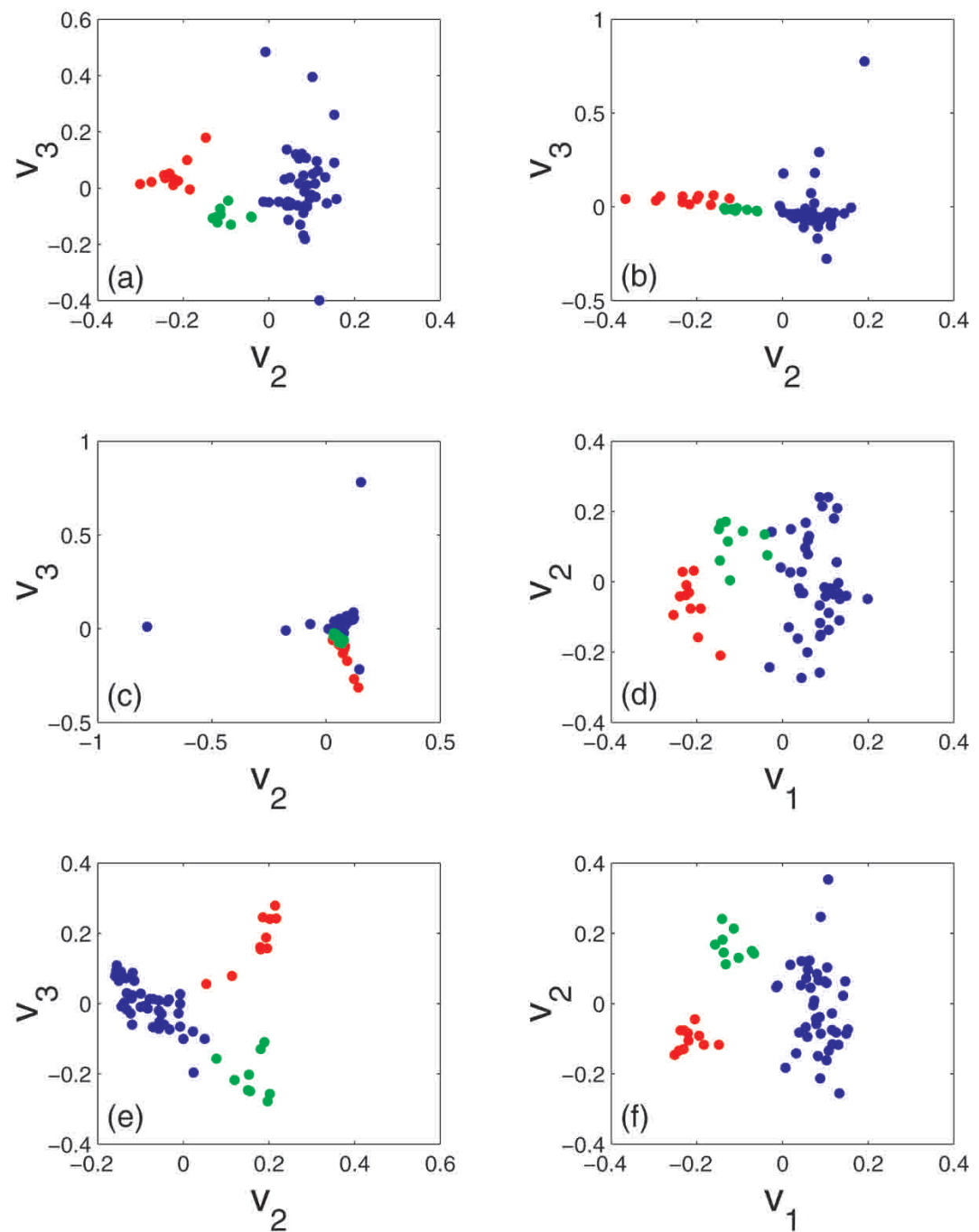

Figure 3 Lymphoma: Scatter plot of experimental conditions of the two best class partitioning eigenvectors $\mathrm{v}_{\mathrm{i}}, \mathrm{v}_{\mathrm{j}}$. The subscripts (i,j) of these eigenvectors indicate their corresponding singular values. CLL samples are denoted by red dots, DLCL by blue dots, and FL by green dots. (a) Bistochastization: the $2 \mathrm{nd}$ and 3rd eigenvectors of $B B^{T}$. (b) Biclustering: the $2 \mathrm{nd}$ and 3rd eigenvectors of $R^{-1} A C^{-1} A^{T}$. (c) SVD: the 2nd and 3rd eigenvectors of $A A^{T}$. (d) Normalization and SVD: the 1st and 2nd eigenvectors of $\bar{A} \bar{A}^{T}$ where $\bar{A}$ is obtained by first dividing each column of $\mathrm{A}$ by its mean and then standardizing each row of the column normalized matrix. (e) Normalized cut algorithm: 2nd and 3rd eigenvectors of the row-stochastic matrix P. P is obtained by first creating a distance matrix $\mathrm{S}$ using Euclidean distance between the standardized columns of $A$, transforming it to an affinity matrix with zero diagonal elements and off diagonal elements defined as $W_{i j}=\exp \left(-\alpha S_{i j}\right) / \max \left(S_{i j}\right)$ and finally normalizing each row sum of the affinity matrix to one. (f) As in (c) but with an SVD analysis of the log interaction matrix $K$ instead of $A$.

and found only moderate levels of correlation. However, if we instead average the expression levels of each gene over all samples of the same tumor type (obtaining vectors with five entries representing the averages of the five types of tumors), the partition of the genes based on correlation between the five-dimensional vectors is more apparent.

This dataset well fits the specifications of our approach, which is geared to finding a "checkerboard-like structure," indicating that for each type of tumor there may be few characteristic subsets of genes that are either upregulated or downregulated. To understand our method (Fig. 1), consider a situation in which an underlying class structure of genes and of experimental conditions exists. We model the data as a composition of blocks, each of which represents a gene-type- condition-type pairing, but the block structure is not immediately evident. Mathematically, the expression level of a specific gene $i$ under a certain experimental condition $j$ can be expressed as a product of three independent factors. The first factor, which we called the hidden base expression level, is denoted by $E_{\mathrm{ij}}$. We assume that the entries of $E$ within each block are constant. The second factor, denoted $\rho_{i}$, represents the tendency of gene $i$ to be expressed under all experimental conditions. The last factor, denoted $\chi_{j}$, represents the overall tendency of genes to be expressed under condition $j$. We assume the microarray expression data to be a noisy version of the product of these three factors.

\section{Independent Rescaling of Genes and Conditions}

We assume that the data matrix $A$ represents an approximation of the product of these three factors, $E_{i j}, \rho_{i}$, and $\chi_{\mathrm{j}}$. Our objective in the simultaneous clustering of genes and conditions is, given $A$, to find the underlying block structure of $E$. Consider two genes, $i$ and $k$, which belong to a subset of similar genes. On average, according to this model, their expression levels under each condition should be related by a factor of $\rho_{i} / \rho_{k}$. Therefore, if we normalize the two rows, $i$ and $k$, in $A$, then on average they should be identical. The similarity between the expression levels of the two genes should be more noticeable if we take the mean of expression levels with respect to all conditions of the same type. This will lead to an eigenvalue problem, as is shown next. Let $R$ denote a diagonal matrix whose elements $r_{i}$ (where $i=1, \ldots, n)$ represent the row sums of $A\left[R=\operatorname{diag}\left(A \cdot 1_{n}\right), 1_{n}\right.$ denotes the $n$ vector $(1, \ldots, 1)]$. Let $\mathrm{u}=\left(u^{1}, u^{2}, \ldots\right.$, $u^{m}$ ) denote a "classification vector" of experimental conditions, so that $u$ is constant over all conditions of the same type. For instance, if there are two types of conditions, then $u^{j}=\alpha$ for each condition $j$ of the first type and $u^{j}=\beta$ for each condition $j$ of the second type. In other words, if we reorder the conditions such that all conditions of the first type appear first, then $u=(\alpha, \ldots, \alpha, \beta, \ldots \beta)$. Then, $v=R^{-1} A u$ is an estimate of a "gene classification vector," that is, a vector whose entries are constant for all genes of the same type (e.g., if there are two types of genes, then $v_{i}=\gamma$ for each gene $i$ of the first type and $v_{i}=\delta$ for each gene $i$ of the second type). By multiplying by $R^{-1}$ from the left. we normalize the rows of $A$, and by applying this normalized matrix to $u$, we obtain a weighted sum of estimates of the mean expression level of every gene $i$ under every type of experimental condition. When a hidden block structure exists for every pair of genes of the same type, these linear combinations are estimates of the same value. 

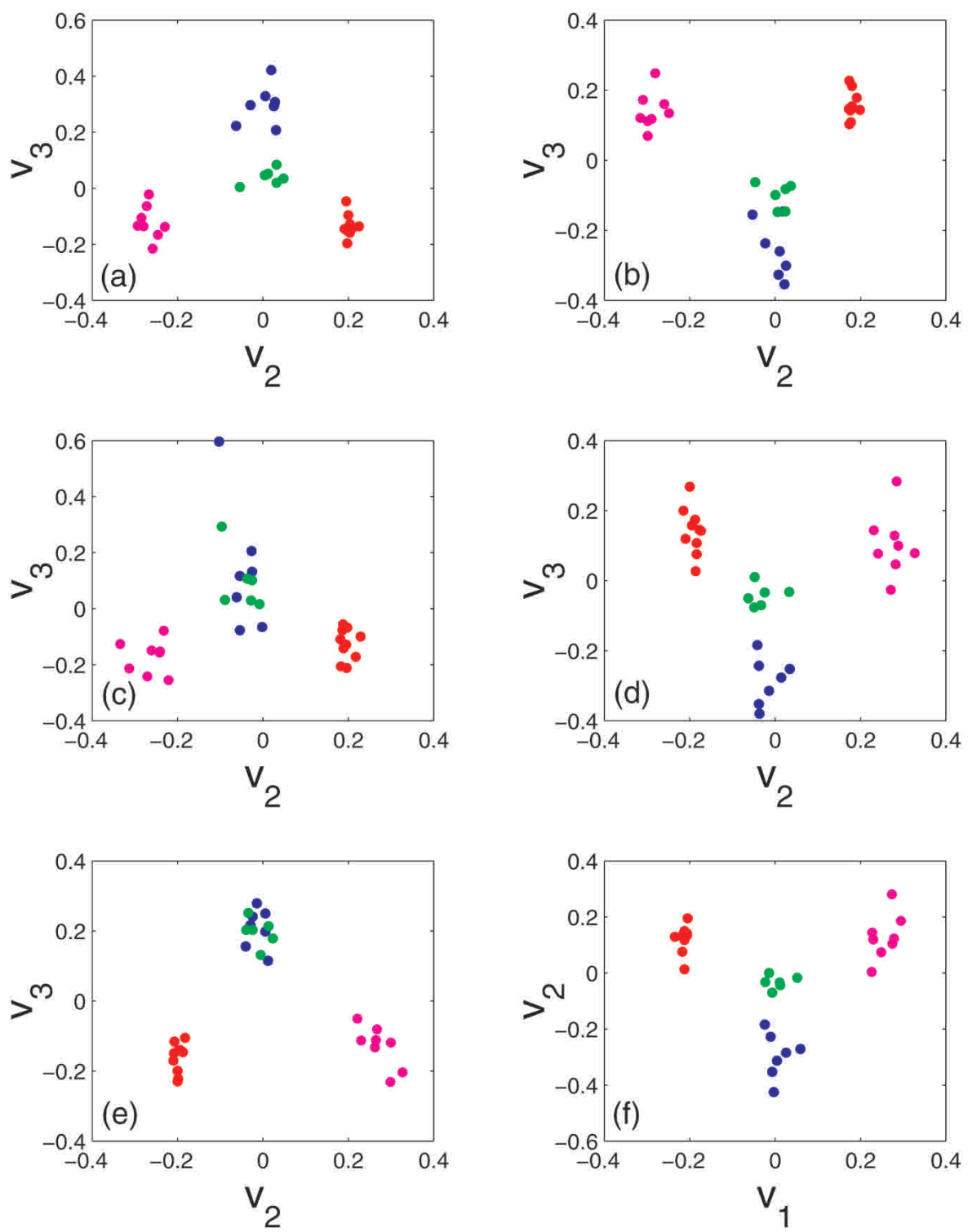

Figure 4 Scatter plots as in Fig. 3 with another lymphoma dataset generated using Affymetrix chips (Klein et al. 2001) instead of microarrays. DLCL samples are denoted by green dots, CLL by blue dots, FL by yellow dots, and DLCL cell lines by magenta dots.

The same reasoning applies to the columns. If we now apply $C^{-1} A^{\mathrm{T}} v$, where $C$ is the diagonal matrix whose components are the column sums of $A\left[C=\operatorname{diag}\left(1_{m}^{T} \cdot A\right)\right]$, we obtain for each experimental condition $j$ a weighted sum of estimates of the mean expression level of genes of the same type. Consequently, the result of applying the matrix $C^{-1} A^{\mathrm{T}} R^{-1} A$ to a condition classification vector, $v$, should also be a condition classification vector. We will denote this matrix by $M_{1} \cdot M_{1}$ has a number of characteristics: it is positive semidefinite, it has only real non-negative eigenvalues, and its dominant eigenvector is $(1 / \sqrt{m}) 1_{m}$ with eigenvalue 1 . Moreover, assuming $E$ has linearly independent blocks, its rank is at least $\min \left(\mathrm{n}_{r}, \mathrm{n}_{c}\right)$, where $\mathrm{n}_{r}$ denotes the number of gene classes and $\mathrm{n}_{c}$ denotes the number of experimental condition classes. (In general the rank would be higher due to noise.) Note that for data with $\mathrm{n}_{c}$ classes of experimental conditions, the set of all classification vectors spans a linear subspace of dimension $\mathrm{n}_{c}$. (This is be- cause a classification vector may have a different constant value for each of the $\mathrm{n}_{c}$ types of experimental conditions.) Therefore, there exists at least one vector that satisfies $M_{1} u=\lambda u$. (In fact, there are exactly $\min \left(\mathrm{n}_{r}, \mathrm{n}_{c}\right)$ such vectors $)$. One of these eigenvectors is the trivial vector $(1 / \sqrt{m}) 1_{m}$. Similarly, there exists at least one gene classification vector that satisfies $M_{2} v=\lambda v$, with $M_{2}=R^{-1} A C^{-1} A^{\mathrm{T}}$. (Note that $M_{1}$ and $M_{2}$ have the same sets of eigenvalues such that if $M_{1} u=\lambda u$ then $M_{2} v=\lambda v$ with $v=R^{-1} A u$.) These classification vectors can be estimated by solving the two eigensystems above. A roughly piecewise constant structure in the eigenvectors indicates the clusters of both genes and conditions in the data.

These two eigenvalue problems can be solved through a standard SVD of the rescaled matrix $\hat{A} \equiv R^{-1 / 2} A C^{-1 / 2}$ realizing that the equation $\hat{A}^{T} \hat{A} w \equiv C^{-1 /}$ $2 A^{T} R^{-1} A C^{-1 / 2} w=\lambda w$ that is used to find the singular values of $\hat{A}$ is equivalent to the above eigenvalue problem $C^{-1} A^{\mathrm{T}} R^{-1} A u=\lambda u$ with $u \equiv C^{-1 / 2} w$ (and similarly $\hat{A} \hat{A}^{T} Z \equiv R^{-1 / 2} A C^{-1} A^{T} R^{-1 / 2} Z=\lambda Z$ implies $v \equiv R^{-1 / 2} Z$ ). The outer product $1_{n} 1_{m}^{T}$, which is a matrix containing only entries of one, is the contribution of the first singular value to the rescaled matrix $\hat{A}$. Thus, the first eigenvalue contributes a constant background to both the gene and the experimental condition dimensions, and therefore its effect should be eliminated. Note that although our method is defined through a product of $A$ and $A^{T}$ it does not imply that we multiply the noise, as is evident from the SVD interpretation.

\section{Simultaneous Normalization of Genes and Conditions}

Because our spectral biclustering approach includes the normalization of rows and columns as an integral part of the algorithm, it is natural to attempt to simultaneously normalize both genes and conditions. As described below, this can be achieved by repeating the procedure described above for independent scaling of rows and columns iteratively until convergence.

This process, which we call bistochastization, results in a rectangular matrix $B$ that has a doubly stochastic-like structure-all rows sum to a constant and all columns sum to a different constant. According to Sinkhorn's theorem, $B$ can then be written as a product $B=D_{1} A D_{2}$ where $D_{1}$ and $D_{2}$ are diagonal matrices (Bapat and Raghavan 1997). Such a matrix $B$ exists under quite general conditions on $A$; for example, it is sufficient for all of the entries in $A$ to be positive. In general, $B$ can be computed by repeated normalization of rows and columns (with the normalizing matrices as $R^{-1}$ and $C^{-1}$ or $R^{-1 / 2}$ and $C^{-1 / 2}$ ). $D_{1}$ and $D_{2}$ then will represent the product of all these normalizations. Fast methods to find $D_{1}$ and $D_{2}$ in- 

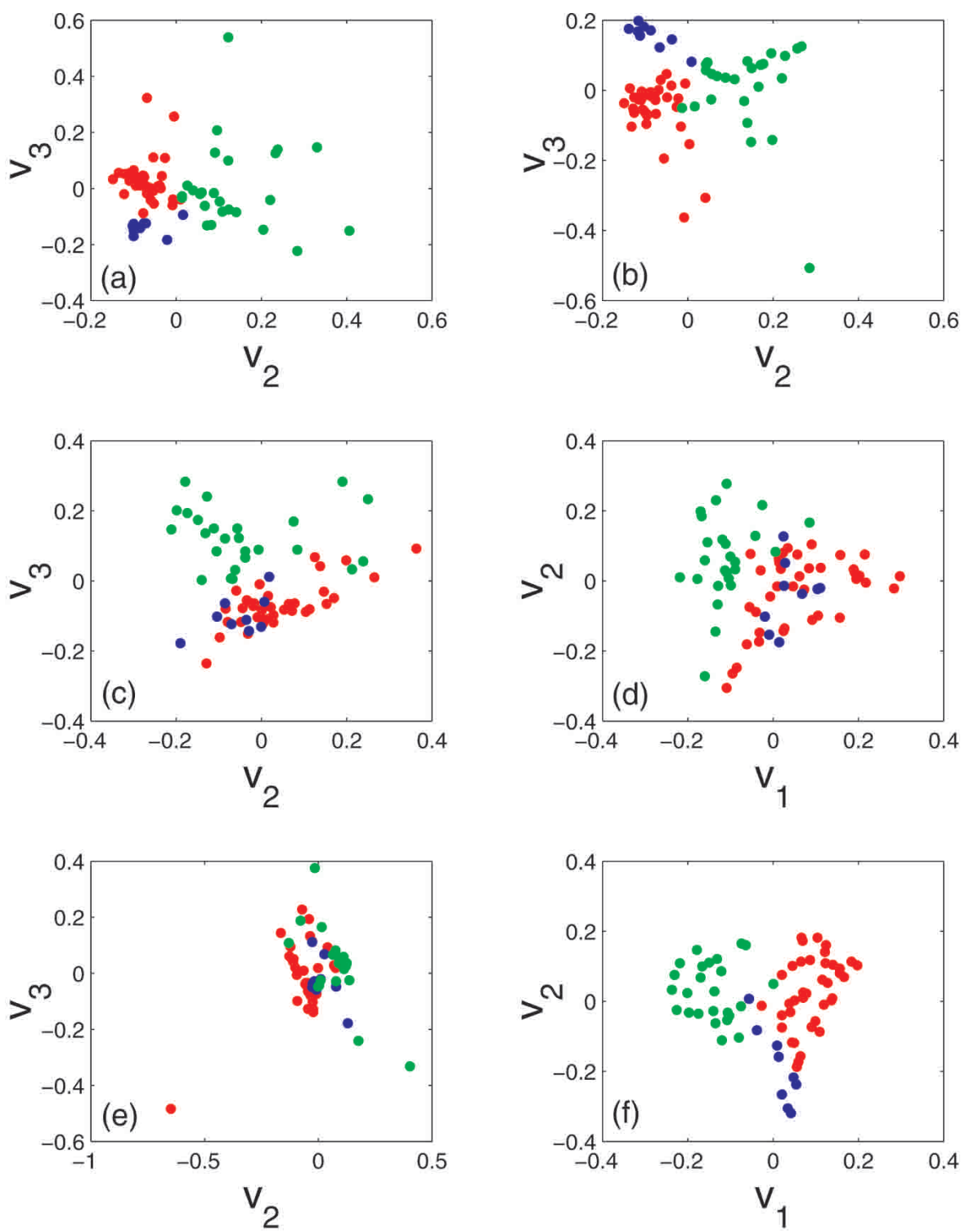

Figure 5 Leukemia data presented in the same format as in Fig. 3. B-cell ALL samples are denoted by red dots, T-cell ALL by blue dots, and AML by green dots. In this analysis we preselected all genes that had positive Affymetrix average difference expression levels.

clude the deviation reduction and balancing algorithms (Bapat and Raghavan 1997). Once $D_{1}$ and $D_{2}$ are found, we apply SVD to $B$ with no further normalization to reveal a block structure.

We have also investigated an alternative to bistochastization that we call the log-interactions normalization. A common and useful practice in microarray analysis is transforming the data by taking logarithms. The resulting transformed data typically have better distributional properties than the data on the original scale-distributions are closer to Normal, scatterplots are more informative, and so forth. The loginteractions normalization method begins by calculating the $\operatorname{logarithm} L_{i j}=\log \left(A_{i j}\right)$ of the given expression data and then extracting the interactions between the genes and the conditions, where the term "interaction" is used as in the analysis of variance (ANOVA).

As above, the log-interactions normalization is motivated by the idea that two genes whose expression profiles differ only by a multiplicative constant of proportionality are really behaving in the same way, and we would like these genes to cluster together. In other words, after taking logs, we would like to consider two genes whose expression profiles differ by an additive constant to be equivalent. This suggests subtracting a constant from each row so that the row means each become 0 , in which case the expression profiles of two genes that we would like to consider equivalent actually become the same. Likewise, the same idea holds for the conditions (columns of the matrix). Constant differences in the log expression profiles between two conditions are considered unimportant, and we subtract a constant from each column so that the column means become 0 . It turns out that these adjustments to the rows and columns of the matrix to achieve row and column means of zero can all be done simultaneously by a simple formula. Defining $\bar{L}_{i .}=(1 / m) \sum_{j=1}^{m} L_{i j}$ to be the average of the $i$ th row, $\bar{L}_{. j}=(1 / n) \sum_{i=1}^{n} L_{i j}$ to be the average of the $j$ th column, and $\bar{L} . .=(1 / m n)$ $\sum_{i=1}^{g} \sum_{j=1}^{m} L_{i j}$ to be the average of the whole matrix, the result of these adjustments is a matrix of interactions $K=\left(K_{i j}\right)$, calculated by the formula $K_{i j}=L_{i j}-\bar{L}_{i .}-\bar{L}_{. j}+\bar{L}_{. . .}$This formula is familiar from the study of twoway ANOVA, from which the terminology of "interactions" is adopted. The interaction $K_{i j}$ between gene $i$ and condition $j$ captures the extra $(\log )$ expression of gene $i$ in condition $j$ that is not explained simply by an overall difference between gene $i$ and other genes or between condition $j$ and other conditions, but rather is special to the combination of gene $i$ with condition $j$. Again, as described before, we apply the SVD to the matrix $K$ to reveal block structure in the interactions. The calculations to obtain the interactions are simpler than bistochastization, as they are done by a simple formula with no iteration. In addition, in this normalization the first singular eigenvectors $u_{1}$ and $v_{1}$ may carry important partitioning information. Therefore we do not automatically discard them as was done in the previously discussed normalizations. Finally, we note another connection between matrices of interactions and matrices resulting from bistochastization. Starting with a matrix of interactions $K$, we can produce a bistochastic matrix simply by adding a constant to $K$.

\section{Postprocessing the Eigenvectors to Find Partitions}

Each of the above normalization approaches (independent scaling, bistochastization, or log interactions) gives rise, after the SVD, to a set of gene and condition eigenvectors (that in the context of microarray analysis are sometimes termed eigengenes and eigenarrays; Hastie et al. 1999; Alter et al. 2000). Now in this section, we deal with the issues of how to interpret these vectors. First recall that in the case of the first 

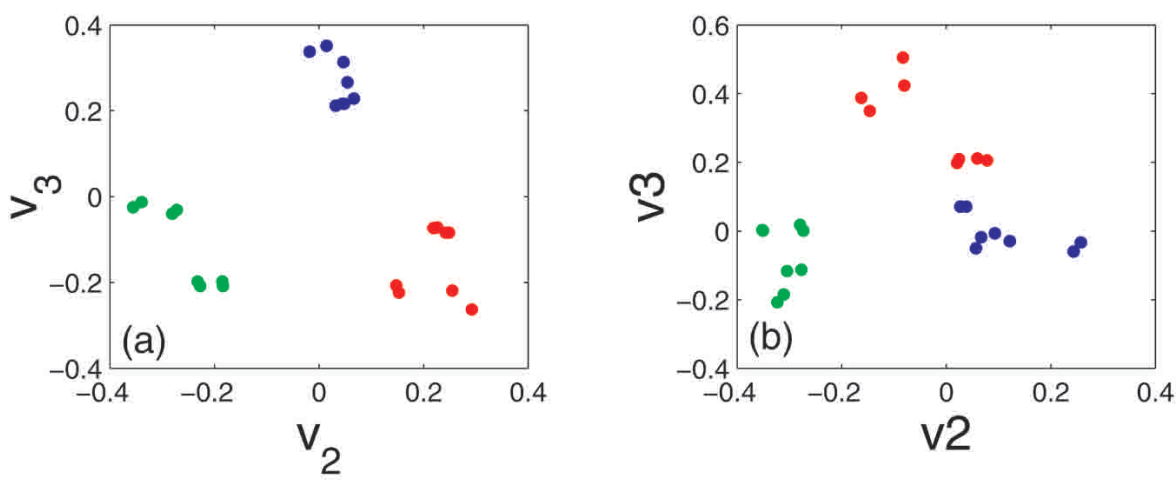

corresponding to the largest eigenvalue would provide the optimal partition in analogy with related spectral approaches to clustering (e.g., Shi and Malik 1997). In principle, the classification eigenvectors may not belong to the largest eigenvalue, and we closely inspect a few eigenvectors that correspond to the first few largest eigenvalues. We observed that for various synthetic data with near-perfect checkerboard-like block structure, the partitioning eigenvectors are commonly associated with one of the largest eigenvalues, but in a few cases an eigenvector with a small
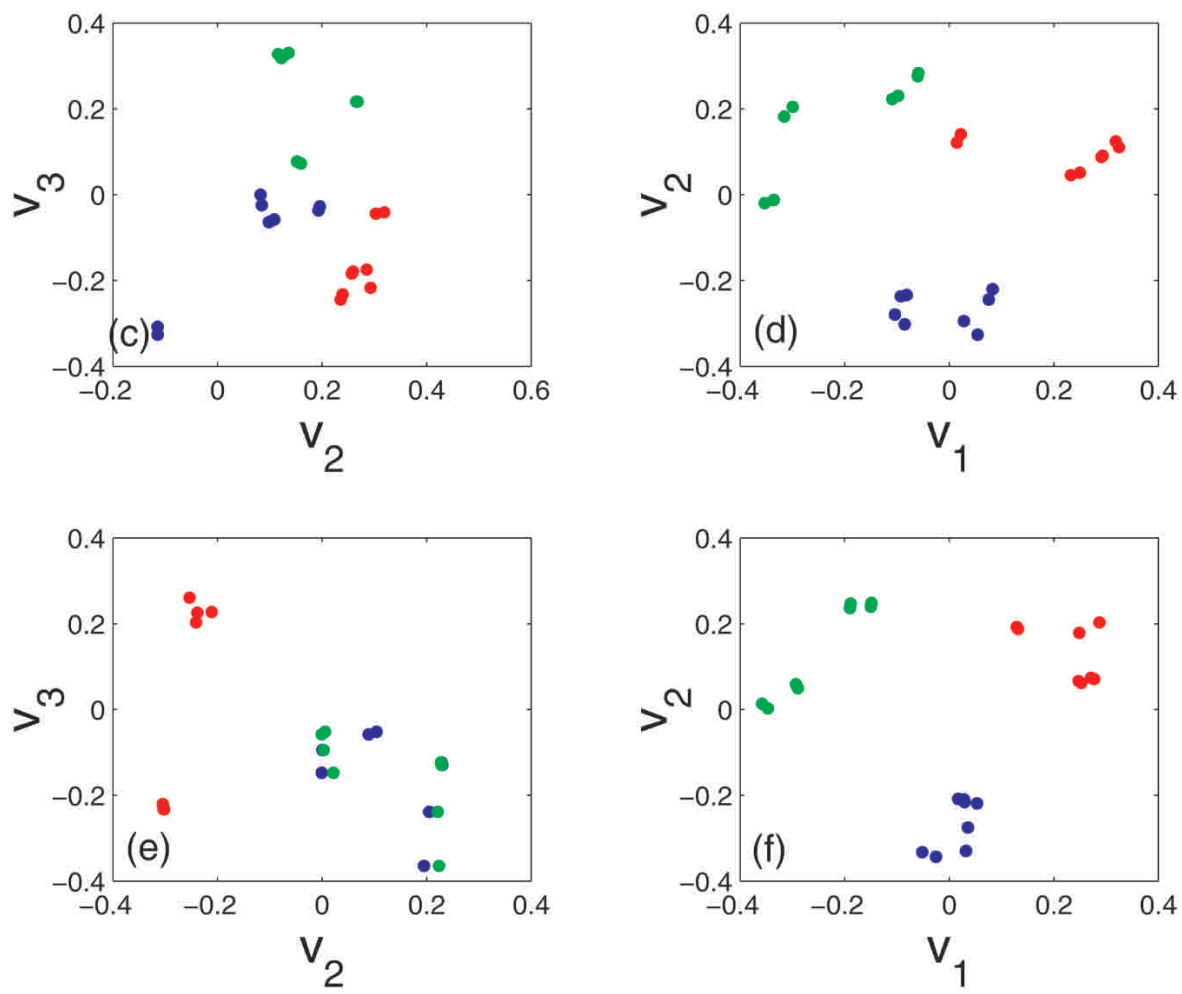

Figure 6 Breast cell lines transfected with the CSF1R oncogene: Scatter plots as in Fig. 3 for mRNA ratios of benign breast cells and wild-type cells transfected with the CSF1R oncogene causing them to invade and metastasize (red), ratios of cells transfected with a mutated oncogene causing an invasive phenotype and cells transfected with the wild-type oncogene (blue), and ratios of cells transfected with a mutated oncogene causing a metastatic phenotype and cells transfected with the wild-type oncogene (green). In this case we preselected differentially expressed genes such that for at least one pair of samples, the genes had a twofold ratio.

two normalizations we discussed (the independent and bistochastic rescaling), we discard the largest eigenvalue, which is trivial in the sense that its eigenvectors make a trivial constant contribution to the matrix, and therefore carry no partitioning information. In the case of the log-interactions normalization, there is no eigenvalue that is trivial in this sense. We will use the terminology "largest eigenvalue" to mean the largest nontrivial eigenvalue, which, for example, is the second largest eigenvalue for the independent and bistochastic normalizations, whereas it is the largest eigenvalue for the log-interactions normalization. If the dataset has an underlying "checkerboard" structure, there is at least one pair of piecewise constant eigenvectors $u$ and $v$ that correspond to the same eigenvalue. One would expect that the eigenvectors eigenvalue could be the partition-

ing one. (This occurs typically when the separation between blocks in $E$ is smaller than the standard deviation within a block.) In order to extract partitioning information from these eigensystems, we examine all the eigenvectors by fitting them to piecewise constant vectors. This is done by sorting the entries of each eigenvector, testing all possible thresholds, and choosing the eigenvector with a partition that is well approximated by a piecewise constant vector. (Selecting one threshold partitions the entries in the sorted eigenvector into two subsets, two thresholds into three subsets, and so forth.) Note that to partition the eigenvector into two, one needs to consider $\mathrm{n}-1$ different thresholds; to partition it into three, it requires inspection of $(n-1)(n-2) / 2$ different thresholds, and so on. This procedure is similar to application of the $\mathrm{k}$-means algorithm to the onedimensional eigenvectors. (In particular, in the experiments below we performed this procedure automatically to the six most dominant eigenvectors.) A common practice in spectral clustering is to perform a final clustering step to the data projected to a small number of eigenvectors, instead of clustering each eigenvector individually (Shi and Malik 1997). In our experiments we too perform a final clustering step by applying both the k-means and the normalized cuts algorithms to the data projected to the best two or three eigenvectors.

Our clustering method provides not only a division into clusters, but also ranks the degree of membership of genes (and conditions) to the respective cluster according to the actual values in the partitioning-sorted eigenvectors. Each partitioning-sorted eigenvector could be approximated by a step-like (piecewise constant) structure, but the values of the sorted eigenvector within each step are monotonically decreasing. These values can be used to rank or represent gradual transitions within clusters. Such rankings may also be useful, for example, for revealing genes related to premalignant conditions, and for studying ranking of patients within a disease cluster in relation to prognosis. 

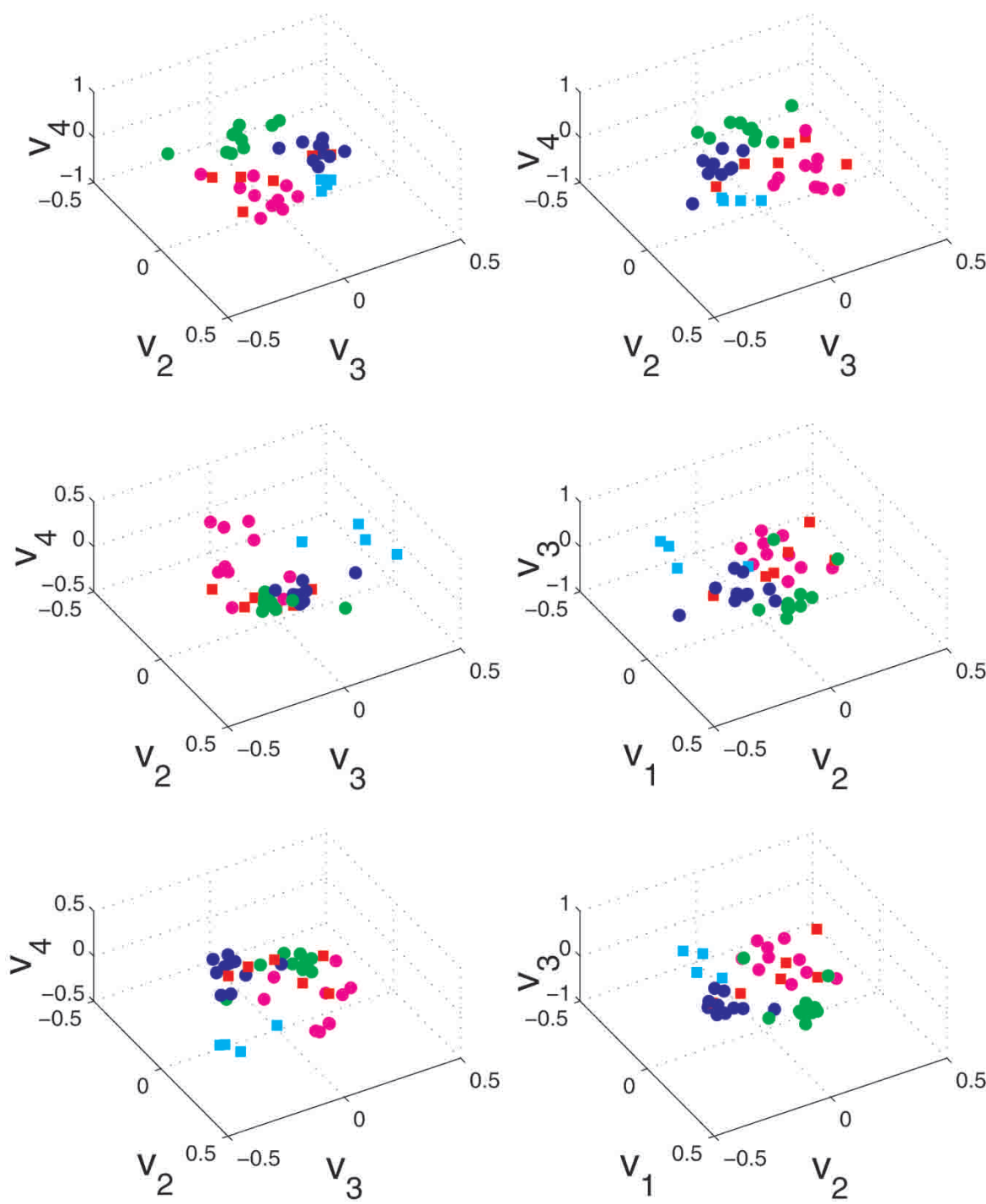

Figure 7 Central nervous system embryonal tumor: Data generated using Affymetrix chips (Pomeroy et al. 2002) of medulloblastoma (blue), malignant glioma (pink), normal cerebella (cyan), rhabdoid (green), and primitive neuro-ectodermal (red) tumors. Scatter plots of experimental conditions projected onto the three best class partitioning eigenvectors using the same format as in Fig. 3.

random noise (Bittner et al. 2000) or subsampling (Ben-Hur et al. 2002) the given data.

We took an alternative approach to assess the quality of a biclustering by testing a null hypothesis of no structure in the data matrix. We first normalized the data and used the best partitioning pair of eigenvectors (among the six leading eigenvectors) to determine an approximate $2 \times 2$ block solution. We then calculated the sum of squared errors (SSE) for the leastsquares fit of these blocks to the normalized data matrix. Finally, to assess the quality of this fit we randomly shuffled the data matrix and applied the same process to the shuffled matrix. For example, in the breast cell oncogene data set described below, fitting the normalized dataset to a $2 \times 2$ matrix obtained by division according to the second largest pair of eigenvectors of the original matrix is compared to fitting of 10,000 shuffled matrices (after bistochastization) to their corresponding best $2 \times 2$ block approximations. The SSE for this dataset is more than 100 standard deviations smaller than the mean of the SSE scores obtained from the shuffled matrices, leading to a correspondingly tiny $P$ value for the hypothesis test of randomness in the data matrix.

\section{Probabilistic Interpretation}

In the biclustering approach, the normalization procedure, obtained by constraining the row sums to be equal to one constant and the column sums to be equal to another constant, is an integral part of the modeling that allows us to discern bidirectional structures. This normalization can be cast in probabilistic terms by imagining first choosing a random RNA transcript from all RNA in all samples (conditions), and then choosing one more RNA transcript randomly from the same

In addition to the uses of biclustering as a tool for data visualization and interpretation, it is natural to ask how to assess the quality of biclusters, in terms of statistical significance, or stability. In general, this type of problem is far from settled; in fact, even in the simpler setting of ordinary clustering new efforts to address these questions regularly continue to appear. One type of approach attempts to quantify the "stability" of suspected structure observed in the given data. This is done by mimicking the operation of collecting repeated independent data samples from the same datagenerating distribution, repeating the analysis on those artificial samples, and seeing how frequently the suspected structure is observed in the artificial data. If the observed data contain sufficient replication, then the bootstrap approach of Kerr and Churchill (2001) may be applied to generate the artificial replicated data sets. However, most experiments still lack the sort of replication required to carry this out. For such experiments, one could generate artificial data sets by adding sample. Here, when we speak of choosing "randomly" we mean that each possible RNA is equally likely to be chosen. Having chosen these two RNAs, we take note of which sample they come from and which genes they express. The matrix entry $\left(R^{-1} A\right)_{i j}$ may be interpreted as the conditional probability $p_{s \mid g}(j \mid i)$ that the sample is $j$, given that the first RNA chosen was transcribed from gene $i$. Similarly, $\left(C^{-1} A^{T}\right)_{j k}$ may be interpreted as the conditional probability that the gene corresponding to the first transcript is $k$, given that the sample is $j$. Moreover, the product of the row-normalized matrix and the column-normalized matrix approximates the conditional probability $p_{g}{ }_{g}(i \mid k)$ of choosing a transcript from gene $i$, given that we also chose one from gene $k$. This is so because, under the assumption that $k$ and $i$ are approximately conditionally independent given $j$, which amounts to saying that the probability of drawing a transcript from gene $k$, conditional on having chosen sample $j$, does not depend on whether or not the other RNA that we drew happened to be from gene $i$, we have

\section{Genome Research}



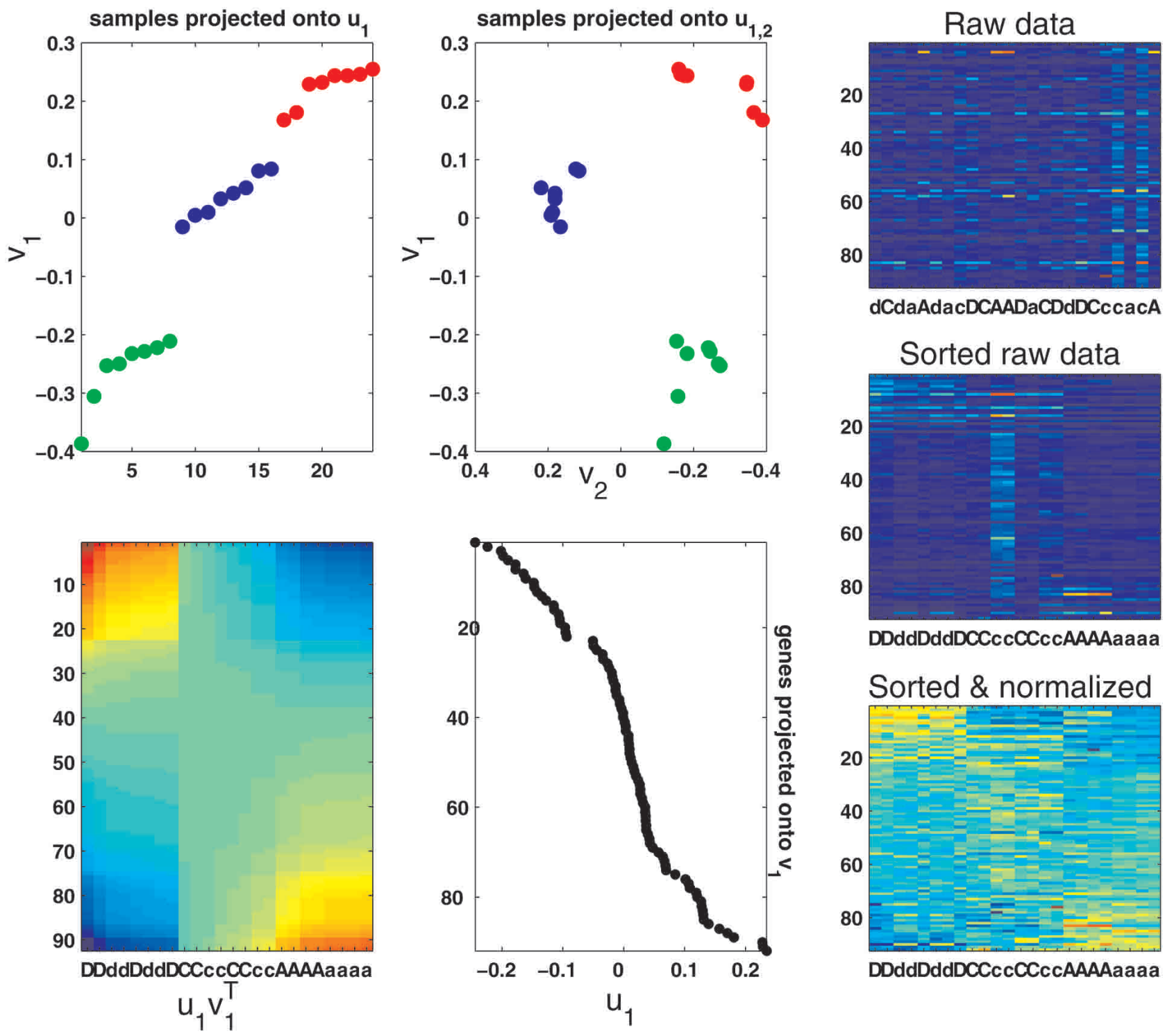

DDddDddDCCccCCccAAAAaaaa

Figure 8 Optimal array partitioning obtained by the 1 st singular vectors of the log-interaction matrix. The data consist of eight measurements of mRNA ratios for three pairs of cell types: $(\mathrm{A}, \mathrm{a})$ benign breast cells and the wild-type cells transfected with the CSF1R oncogene causing them to invade and metastasize; $(C, C)$ cells transfected with a mutated oncogene causing an invasive phenotype and cells transfected with the wild-type oncogene; and (D,d) cells transfected with a mutated oncogene causing a metastatic phenotype and cells transfected with the wild-type oncogene. In this case we preselected differentially expressed genes such that for at least one pair of samples, the genes had a threefold ratio. The sorted eigen-gene $v_{1}$ and eigen-array $u_{1}$ have gaps indicating partitioning of patients and genes, respectively. As a result, the outer product matrix $\operatorname{sort}\left(u_{7}\right) \operatorname{sort}\left(v_{7}\right)^{\top}$ has a "soft" block structure. The block structure is hardly seen when the raw data are sorted but not normalized. However, it is more noticeable when the data are both sorted and normalized. Also shown are the conditions projected onto the first two partitioning eigenvectors $\mathrm{u}_{1}$ and $\mathrm{u}_{2}$. Obviously, using the extra dimension gives a clearer separation.

$$
\begin{aligned}
p_{g \mid g}(k \mid i) & =\sum_{j} p_{s \mid g}(j \mid i) p_{g \mid s g}(k \mid j, i) \approx \sum_{j} p_{s \mid g}(j \mid i) p_{g \mid s}(k \mid j) \\
& =\left[\left(R^{-1} A\right)\left(C^{-1} A^{T}\right)\right]_{i k} .
\end{aligned}
$$

This expression reflects the tendency of genes $i$ and $k$ to coexpress, averaged over the different samples. Similarly, the product of the column and row-normalized matrices approximates the conditional probability $p_{s \mid s}(j \mid l)$ that reflects the similarity between the expression profiles of samples $j$ and $l$. Note that the probabilities $p_{g \mid g}(i \mid k)$ and $p_{s \mid s}(j \mid l)$ define asymmetrical affinity measures between any pair $(i, k)$ of genes and any pair $(j, l)$ of samples, respectively. This is very different from the usual symmetrical affinity measures, for example, correlation, used to describe the relationship between genes. However, for bistochastizaton, the matrices $B^{T} B$ and $B B^{T}$ represent symmetrical affinities, $p_{g \mid g}(i \mid k)=p_{g \mid g}(k \mid i)$ and $p_{s \mid s}(j \mid l)=p_{s \mid s}(l \mid j)$, respectively.

\section{RESULTS}

Overall Format of the Results

We have performed a study in which we applied the above spectral biclustering methods to five groups of cancer micro- 
array data sets-lymphoma (microarray and Affymetrix), leukemia, breast cancer, and central nervous system embryonal tumors. As explained above, we utilized SVD to find pairs of piecewise constant eigenvectors of genes and conditions, that reflect the degree to which the data can be rearranged in a checkerboard structure. Our methods employ specific normalization schemes that highlight the similarity of both gene and condition eigenvectors to piecewise constant vectors, and this similarity, in turn, directly reflects the degree of biclustering. To assess our procedure, it is useful to see how well it compares to several benchmarks, with respect to achieving the goal of piecewise constant eigenvectors.

Our main results are presented in Figures 3-7. These show consistently formatted graphs of the projection of each dataset onto the best two eigenvectors. Each figure is laid out in six panels, with the first two panels associated with our biclustering methods and the next four panels showing the benchmarks. In particular:

Panel $a$ Bistochastization shows biclustering using the bistochastic normalization.

Panel $b$ Biclustering shows standard biclustering with independent rescaling of rows and columns.

Panel $c$ SVD shows SVD applied to the raw data matrix $A$.

Panel $d$ Binormalization shows SVD applied to a transformed matrix obtained by first rescaling its columns by their means and then standardizing the rows of the rescaled matrix as proposed in Getz et al. (2000).

Panel $e$ Normalized cuts shows a normalized cuts benchmark. Here we apply the normalized cuts algorithm using an affinity matrix obtained from a distance matrix, which, in turn, was derived by calculating the norms of the differences between the standardized columns of $A$ as proposed in Xing and Karp (2001). (See caption of Fig. 3 for more details.) Moreover, we applied the normalized cuts algorithm to an affinity matrix constructed from the column-rescaled rowstandardized matrix (Getz et al. 2000), as in panel (d). We then examined whether a partition is visible in the eigenvectors that correspond to the second largest eigenvalue (which in the normalized cuts case are supposed to provide approximation of the optimal partition) and in the subspace spanned by two or three eigenvectors with the best proximity to piecewise constant vectors.

Panel $f$ Log-interaction shows SVD applied to a matrix where the raw expression data is substituted by the matrix $K$ described above.

Overall, by comparing the six panels in each of the five different figures, we see that in the bistochastization method (panel $a$ ) the distributions of the different samples have no or minimal overlap between clusters as well as more tendency to result in more compact clusters. The biclustering method (panel $b$ ) results in slightly less separable clusters, but it tends to separate the clusters along a single eigenvector. Straight SVD of the different raw data (panel $c$ ) underperforms in comparison to our spectral methods, as can be seen from the intermingled distributions of tumors of different types or less distinct clusters. Performing instead SVD on the loginteraction matrix of the raw expression data tends to produce results that are similar to those obtained with bistochastization (panel $f$ ). SVD of the column-rescaled rowstandardized matrix (Getz et al. 2000) and the normalized cut method result in better partitioning than SVD of the raw data (panels $d$ and $e$ ). However, in general, our spectral methods consistently perform well.

In the following sections we discuss each of the five datasets in detail.

\section{Lymphoma Microarray Dataset}

We first applied the methods to publicly available lymphoma microarray data: chronic lymphocytic leukemia (CLL), diffuse large B-cell lymphoma (DLCL), and follicular lymphoma (FL). The clustering results are shown in Figures 2 and 3. In both cases when we used the doubly stochastic-like matrix $B$ or the biclustering method $\left(C^{-1} A^{\mathrm{T}} R^{-1} A\right)$ of the lymphoma dataset, we obtained the desired partitioning of patients in the second largest eigenvectors. The sorted eigenvectors give not only a partition of patients, but also an internal ranking of patients within a given disease. In addition, the outer product of the gene and tumor (sorted) eigenvectors allows us to observe which genes induce a partition of patients and vice versa. This can be seen in Figure 2. Dividing the eigenvector that corresponds to the second largest eigenvalue (in both methods) using the k-means algorithm (which is equivalent to fitting a piecewise constant vector to each of the eigenvectors) led to a clean partition between the DLCL patients and the patients with other diseases. This is highlighted in the header of Figure 2 and the $x$-axis of Figure 3a,b. The published analysis did not cluster two of the DLCL cases correctly (Alizadeh et al. 2000). Further partitioning of the CLL and the FL patients is obtained by using both the second- and third-largest eigenvectors. To divide the data we applied a recursive, two-way clustering using the normalized cuts algorithm to a two-column matrix composed of the 2nd and 3rd eigenvectors of both matrices. (Performing a final clustering step to the data projected to a small number of eigenvectors is a common practice in spectral clustering.) Using the biclustering matrix with independent row and column normalizations, the patients were correctly divided, with the exception of two of the CLL patients, who were clustered together with the FL patients. The best partition was obtained using our doubly stochastic matrix that divided the patients perfectly according to the three types of diseases.

\section{Lymphoma Affymetrix Dataset}

The above lymphoma data were generated by microarray technology that provides relative measurements of expression data. We repeated the lymphoma analysis using data from a study relating B-CLL to memory B cells (Klein et al. 2001). These data were generated using Affymetrix U95A gene chips, which presumably allow measurements proportional to absolute mRNA levels. We selected samples taken from CLL, FL, and DLCL patients, but in addition we also included samples from DLCL cell lines. As can be seen in Figure $4 a$,b, the bistochastization method cleanly separates the four different sample types, and the biclustering separates these samples except for one DLCL sample that slightly overlaps with the FL distribution. We note that the DLCL patient expression patterns are closer to those of the FL patients than to the expression profiles of the DLCL cell lines (and $p_{g \mid g}($ DLCL $\mid \mathrm{FL})>p_{g \mid g}($ DLCL $\mid$ DLCL-cell lines $)$.

\section{Leukemia Dataset}

We applied our methods to public microarray data of acute leukemia (B- and T-cell acute lymphocytic leukemia [ALL] and acute myelogenous leukemia [AML]). The patient distribu-

\section{Genome Research}


tions of the different diseases of the leukemia dataset become separated in the two-dimensional graphs generated by projecting the patient expression profiles onto the 2nd and 3rd gene class partition vectors of the biclustering method (Fig. $5 b)$. The bistochastic method also partitions the patients well, with only one ambiguous case that is close to the boundary between ALL and AML (Fig. 5a). Application of k-means to a matrix composed of the 2 nd and 3rd biclustering eigenvectors results in three misclassifications, which is a slight improvement over the four misclassifications reported by Golub et al. (1999). Further partitioning of the ALL cases is obtained by applying a normalized cuts clustering method to the biclustering eigenvectors, and produces a clear separation between T- and B-cell ALL. This is a slight improvement over published results (two misclassifications; Golub et al. 1999; Getz et al. 2000). Another advantage over their methods is that biclustering does not require specification of the number of desired clusters or lengthy searches for subsets of genes.

\section{Dataset From Breast Cell Lines Transfected With the CSFIR Oncogene}

In another microarray experiment study (Kluger et al. 2001), an oncogene encoding a transmembrane tyrosine kinase receptor was mutated at two different phosphorylation sites. Benign breast cells were transfected with the wild-type oncogene, creating a phenotype that invades and metastasizes. The benign cell line was then transfected with the two mutated oncogenes, creating one phenotype that invades and another one that metastasizes. RNA expression levels were measured eight times for each phenotype. Transfection with a single oncogene is expected to generate similar expression profiles, presumably because only a few genes are biologically influenced. Therefore, it was desirable to see whether profiles of the different phenotypes can be partitioned.

Figure 8 allows us to examine the extent to which the data can be arranged in a checkerboard pattern. This is done by taking the outer product of the cell type-sorted eigenvector that has the most stepwise-like structure (and is associated with the first largest singular value) with the corresponding gene-sorted eigenvector. Due to noise in the data and similarity between the different samples, common clustering techniques such as hierarchical, k-means, and medoids did not succeed in cleanly partitioning the data, but the relevant eigen-array obtained following bistochastization or loginteraction normalization partitioned the samples perfectly. Expression levels of the four cell lines were measured in two separate sets of four measurements. We chose to measure the ratio of three of the cell lines: benign $(a)$, invasive $(c)$, and metastatic $(d)$ with respect to the cell line that invades and metastasizes (b) in the first batch, and the corresponding ratios were similarly derived for the second batch. In Figure 8, the ratios from the first and second batches are denoted by $(a$, $c, d)$ and $(A, C, D)$, respectively. As can be seen, the simultaneous normalization methods partition the data such that all the phenotypes are separated into clusters-that is, " $a$ "s were clustered with " $A$ "s in one group, " $c$ "s with " $C$ "s in another group, and " $d$ "s with " $D$ "s in yet another group, as expected. Further exploration is required in order to relate those gene clusters to biological pathways that are relevant to these conditions.

\section{Central Nervous System Embryonal Tumor Dataset}

Finally, we analyzed the recently published CNS embryonal tumor dataset (Pomeroy et al. 2002): Pomeroy et al. parti- tioned these five tumor types using standard principal component analysis, but did so after employing a preselection of genes exhibiting variation across the data set (see Fig. 1b in Pomeroy et al. 2002). Using all genes, we find that the bistochastization method, and to a lesser degree the biclustering method, partitioned the medulloblastoma, malignant glioma, and normal cerebella tumors. As can be seen in Figure 7, the remaining rhabdoid tumors are more widely scattered in the subspace obtained by projecting the tumors onto the 2 nd -4 th gene partitioning eigenvectors of the biclustering and bistochastization methods. Nonetheless, the rhabdoid tumor distribution does not overlap with the other tumor distributions if we use the bistochastization method. The primitive neuroectodermal tumors (PNETs) did not cluster and were difficult to classify even using supervised methods.

\section{DISCUSSION}

Unsupervised clustering of genes and experimental conditions in microarray data can potentially reveal genes that participate in cellular mechanisms that are involved in various diseases. In this paper we present a spectral biclustering method that utilizes the information gained by clustering the conditions to facilitate the clustering of genes, and vice versa. The method incorporates a closely integrated normalization. It also naturally discards the irrelevant constant background, such that no additional arguments are needed to ignore the contribution associated with the largest eigenvalue, as advocated in Alter et al. (2000). In particular, our method is designed to cluster populations of different tumors assuming that each tumor type has a subset of marker genes that exhibit overexpression and that typically are not overexpressed in other tumors. The main underlying assumption is that we can simultaneously obtain better tumor clusters and gene clusters by correlating genes averaged over different samples of the same tumors. Likewise, the correlation of two tumors is more apparent when averaged over sets of genes of similar expression profiles. In situations where the number of tumor types (the number of clusters of experimental conditions) happens to be equal to the number of typical gene profiles (the number of gene clusters), the biclustering algorithm is related to the modified normalized cuts objective function introduced by Dhillon (2001). In addition, in a situation where the data have approximately a checkerboard structure with more than two clusters on each side, there may be several eigenvectors indicating a partitioning. In this case we may be able to determine the number of clusters by identifying all of these eigenvectors, for example, using a pairwise measure such as mutual entropy between all pairs of eigenvectors.

The methods presented in this paper, particularly those incorporating simultaneous normalization of rows and columns, show consistent advantage over SVD spectral analysis of the raw data, the logarithm of the raw data, other forms of rescaling transformations of the raw data, and the normalized cuts partitioning of the raw or rescaled data. Nevertheless, our partitioning results are not perfect. Better results may be obtained by employing a generative model that better suits the data. It has been shown that removal of irrelevant genes that introduce noise can further improve clustering (as in Xing and Karp 2001). Furthermore, if partitioning in the gene dimension is sharper than partitioning in the condition dimension or vice versa, we can organize the conditions or genes of the blurrier dimension contiguously. Such arrangements perhaps give one a sense of the progression of disease states or relevance of a gene to a particular disease. 


\section{ACKNOWLEDGMENTS}

Y.K. is supported by the Cancer Bioinformatics Fellowship from the Anna Fuller Fund, and M.G. acknowledges support from Human Genome array: Technology for Functional Analysis (an NIH grant number P50 HG02357-01).

The publication costs of this article were defrayed in part by payment of page charges. This article must therefore be hereby marked "advertisement" in accordance with 18 USC section 1734 solely to indicate this fact.

\section{REFERENCES}

Alizadeh, A.A., Eisen, M.B., Davis, R.E., Ma, C., Lossos, I.S Rosenwald, A., Boldrick, J.C., Sabet, H., Tran, T., Yu, X., et al. 2000. Distinct types of diffuse large B-cell lymphoma identified by gene expression profiling. Nature 403: 503-511.

Alter, O., Brown, P.O., and Botstein, D. 2000. Singular value decomposition for genome-wide expression data processing and modeling. Proc. Natl. Acad. Sci. 97: 10101-10106.

Bapat, R.B. and Raghavan, T.E.S. 1997. Non-negative matrices and applications. Chapter 6. Cambridge University Press, Cambridge, UK.

Ben-Hur, A., Elisseeff, A., and Guyon, I. 2002. A stability based method for discovering structure in clustered data. Pac. Symp. Biocomput:: 6-17.

Bittner, M., Meltzer, P., Chen, Y., Jiang, Y., Seftor, E., Hendrix, M., Radmacher, M., Simon, R., Yakhini, Z., Ben-Dor, A., et al. 2000. Molecular classification of cutaneous malignant melanoma by gene expression profiling. Nature 406: 536-540.

Brown, P.O. and Botstein, D. 1999. Exploring the new world of the genome with DNA microarrays. Nat. Genet. 21: 33-37.

Brown, M.P.S., Grundy, W.N., Lin, D., Sugnet, C., Ares, J.M., and Haussler, D. 2000. Knowledge-based analysis of microarray gene expression data by using support vector machines. Proc. Natl. Acad. Sci. 97: 262-267.

Cheng, Y. and Church, G.M. 2000. Biclustering of expression data In $8^{\text {th }}$ International Conference on Intelligent Systems for Molecular Biology, August 2000. UC San Diego, La Jolla, CA

Dhillon, I.S. 2001. Coclustering documents and words using bipartite spectral graph partitioning. In Proceedings of the Seventh Association for Computing Machinery, Special Interest Group on Knowledge Discovery in Data and Data Mining Conference, San Francisco, CA.

Eisen, M., Spellman, P.T., Brown, P.O., and Botstein, D. 1998. Cluster analysis and display of genome-wide expression patterns. Proc. Natl. Acad. Sci. 95: 14863-14868.

Friedman, N., Linial, M., Nachman, I., and Pe'er, D. 2000. Using Bayesian networks to analyze expression data. J. Comp. Biol. 7: 601-620.

Getz, G., Levine, E., and Domany, E. 2000. Coupled two-way clustering analysis of gene microarray data. Proc. Natl. Acad. Sci. 97: 12079-12084

Golub, G.H. and Van Loan, C.F. 1983. Matrix computations. Johns Hopkins University Press, Baltimore, MD.

Golub, T.R., Slonim, D.K., Tamayo, P., Huard, C., Gaasenbeek, M., Mesirov, J.P., Coller, H., Loh, M., Downing, J.R., Caligiuri, M., et al. 1999. Molecular classification of cancer: Class discovery and class prediction by gene expression monitoring. Science 286: $531-537$.

Hartigan, J.A. 1972. Direct clustering of a data matrix. J. Am. Stat. Assoc. 67: 123-129.

Hastie, T., Tibshirani, R., Eisen, M.B., Alizadeh, A., Levy, R., Staudt, L., Chan, W.C., Botstein, D., and Brown, P.O. 2000. "Gene shaving" as a method for identifying distinct sets of genes with similar expression patterns. Genome Biol. 1: research0003.00010003.0021

Hastie, T., Tibshirani, R., Sherlock, G., Eisen, M., Brown, P.O., and Botstein, D. 1999. Imputing missing data for gene expression arrays. Stanford Statistics Department, Stanford, CA. http://www-stat.stanford.edu/ hastie/papers/missing.pdf

Hofmann, T. and Puzicha, J. 1999. Latent class models for collaborative filtering. In Proceedings of the International Joint
Conference in Artificial Intelligence. IJCAI 1999: pp. 688-693. IJCAI Inc., Somerset, NJ.

Holter, N.S., Mitra, M., Maritan, A., Cieplak, M., Banavar, J.R., and Fedoroff, N.V. 2000. Fundamental patterns underlying gene expression profiles: Simplicity from complexity. Proc. Natl. Acad. Sci. 97: 8409-8414.

Kerr, M.K. and Churchill, G.A. 2001. Bootstrapping cluster analysis: Assessing the reliability of conclusions from microarray experiments. Proc. Natl. Acad. Sci. 98: 8961-8965.

Klein, U., Tu, Y., Stolovitzky, G.A., Mattioli, M., Cattoretti, G., Husson, H., Freedman, A., Inghirami, G., Cro, L., Baldini, L., et al. 2001. Gene expression profiling of B cell chronic lymphocytic leukemia reveals a homogeneous phenotype related to memory B cells. J. Exp. Med. 194: 1625-1638.

Kluger, H., Kacinski, B., Kluger, Y., Mironenko, O., Gilmore-Hebert, M., Chang, J., Perkins, A.S., and Sapi, E. 2001. Microarry analysis of invasive and metastatic phenotypes in a breast cancer model. In Poster presented at the Gordon Conference on Cancer, Newport, RI.

Lazzeroni, L. and Owen, A. 2002. Plaid models for gene expression data. Statistica Sinica 12: 61-86.

Lian, Z., Wang, L., Yamaga, S., Bonds, W., Beazer-Barclay, Y., Kluger, Y., Gerstein, M., Newburger, P.E., Berliner, N., and Weissman, S.M. 2001. Genomic and proteomic analysis of the myeloid differentiation program. Blood 98: 513-524.

Lockhart, D.J. and Winzeler, E.A. 2000. Genomics, gene expression and DNA arrays. Nature 405: 827-836.

Mateos, A., Dopazo, J., Jansen, R., Tu, Y., Gerstein, M., and Stolovitzky, G. 2002. Systematic learning of gene functional classes from DNA array expression data by using multilayer perceptrons. Genome Res. 12: 1703-1715.

Pearson, K. 1901. On lines and planes of closest fit to systems of points in space. The London, Edinburgh and Dublin Philosophical Magazine and Journal of Science, Sixth Series 2: 559-572.

Pomeroy, S.L., Tamayo, P., Gaasenbeek, M., Sturla, L.M., Angelo, M., McLaughlin, M.E., Kim, J.Y., Goumnerova, L.C., Black, P.M., Lau, C., et al. 2002. Prediction of central nervous system embryonal tumour outcome based on gene expression. Nature 415: $436-442$.

Raychaudhuri, S., Stuart, J.M., and Altman, R.B. 2000. Principal components analysis to summarize microarray experiments: Application to sporulation time series. In 2000 Pacific Symposium on Biocomputing, pp. 452-463.

Shi, J. and Malik, J. 1997. Normalized cuts and image segmentation. In IEEE Conf. Computer Vision and Pattern Recognition, pp. 731-737.

Stolovitzky, G., Califano, A., and Tu, Y. 2000. Analysis of gene expression microarrays for phenotype classification. In $8^{\text {th }}$ International Conference on Intelligent Systems for Molecular Biology, August 2000. UC San Diego, La Jolla, CA.

Tamayo, P., Slonim, D., Mesirov, J., Zhu, Q., Kitareewan, S., Dmitrovsky, E., Lander, E.S., and Golub, T.R. 1999. Interpreting patterns of gene expression with self-organizing maps: Methods and application to hematopoietic differentiation. Proc. Natl. Acad. Sci. 96: 2907-2912.

Ungar, L. and Foster, A. 1998. A formal statistical approach to collaborative filtering. In Conference on Automated Learning and Discovery CONALD '98, CMU.

van't Veer, L.J., Dai, H., van de Vijver, M.J., He, Y.D., Hart, A.A., Mao, M., Peterse, H.L., van der Kooy, K., Marton, M.J. Witteveen, A.T., et al. 2002. Gene expression profiling predicts clinical outcome of breast cancer. Nature 415: 530-536.

Weiss, Y. 1999. Segmentation using eigenvectors: A unifying view. In Proceedings IEEE International Conference on Computer Vision, pp. 975-982.

Xing, E.P. and Karp, R.M. 2001. CLIFF: Clustering of highdimensional microarray data via iterative feature filtering using normalized cuts. In $9^{\text {th }}$ International Conference on Intelligent Systems for Molecular Biology, July 2001. Copenhagen, Denmark.

Received July 22, 2002; accepted in revised form January 28, 2003 


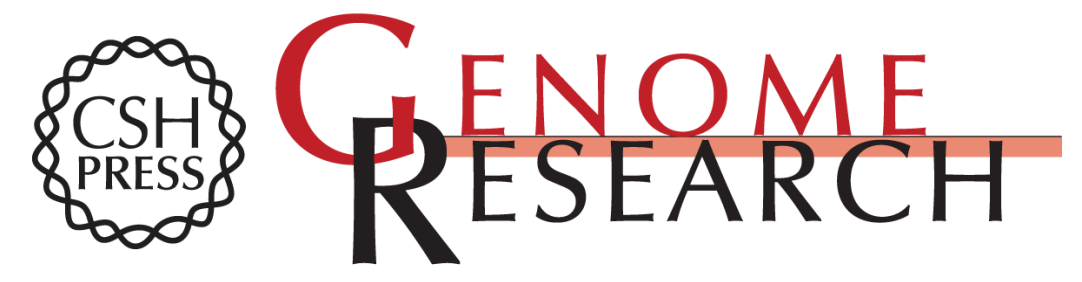

\section{Spectral Biclustering of Microarray Data: Coclustering Genes and Conditions}

Yuval Kluger, Ronen Basri, Joseph T. Chang, et al.

Genome Res. 2003 13: 703-716

Access the most recent version at doi:10.1101/gr.648603

References This article cites 21 articles, 11 of which can be accessed free at:

http://genome.cshlp.org/content/13/4/703.full.html\#ref-list-1

\section{License}

Email Alerting Receive free email alerts when new articles cite this article - sign up in the box at the Service top right corner of the article or click here.

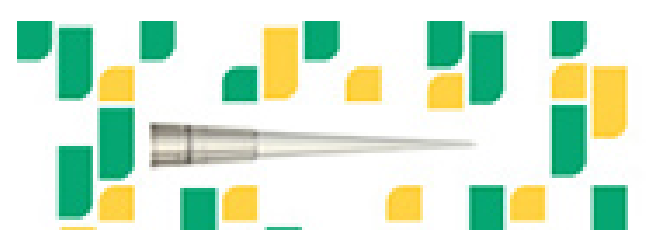

To subscribe to Genome Research go to: https://genome.cshlp.org/subscriptions 